\title{
Inequidades sociales en la progresión de la COVID-19 en población mexicana
}

\author{
Luis Ortiz-Hernández y Miguel A. Pérez-Sastré2
}

Forma de citar Ortiz-Hernández L, Pérez-Sastré MA. Inequidades sociales en la progresión de la COVID-19 en población mexicana. Rev Panam Salud Publica. 2020;44:e106. https://doi.org/10.26633/RPSP.2020.106

RESUMEN

Objetivo. Determinar si existen disparidades asociadas a la estratificación socioeconómica, la etnicidad, los servicios médicos y la región geográfica en la progresión de la enfermedad por coronavirus (COVID-19) en adultos mexicanos infectados por SARS-CoV-2.

Método. Se analizaron los datos registrados por la Dirección General de Epidemiología de la Secretaría de Salud del Gobierno Federal de México respecto de los casos confirmados de infección por SARS-CoV-2. El análisis se limitó a los datos de adultos de 20 años o más registrados hasta el 10 de julio del 2020 ( $n=234$ 870). Los indicadores de severidad de la COVID-19 fueron hospitalización, desarrollo de neumonía, requerimiento de intubación o ingreso a la unidad de cuidados intensivos, y muerte. Se estimaron diferencias de acuerdo con el nivel de marginación municipal, la pertenencia a un grupo indígena, la región geográfica y el sector de los servicios. A partir de modelos de regresión multinivel se estimaron razones de prevalencias (RP). Resultados. Las personas que residían en municipios con mayor marginación tuvieron mayor riesgo de presentar los cuatro indicadores de formas severas de COVID-19 (RP=1,05 o 1,06). Las personas indígenas tuvieron mayor riesgo de neumonía $(R P=1,22)$, hospitalización $(R P=1,14)$ y muerte $(R P=1,23)$. Entre los casos atendidos en servicios privados fue menor el riesgo de muerte $(\mathrm{RP}=0,40)$, pero mayor el uso de intubación o ingreso a la unidad de cuidados intensivos ( $R P=4,45)$.

Conclusiones. Las tendencias observadas indican que los efectos de la COVID-19 no solo se deben a las características biológicas del SARS-CoV-2, sino también a los recursos (o falta de ellos) para enfrentarlo, los cuales están distribuidos por procesos sociales.

Palabras claves Inequidad social; infecciones por coronavirus; factores socioeconómicos; disparidades en el estado de salud; grupos étnicos; México.

Por su rápida diseminación y efectos en la salud, la pandemia de la infección por el coronavirus SARS-CoV-2 es una prioridad mundial. Sociedades y gobiernos están interesados en identificar la forma de reducir las formas graves de la enfermedad por coronavirus (COVID-19), ya que cuando estas se presentan en gran número, los servicios médicos se saturan, lo cual incrementa la mortalidad. En las formas graves de COVID-19 existe mayor riesgo de complicaciones como neumonía, que requiere internamiento y, en algunos casos, soporte de vida. Las formas severas de COVID-19 también presentan mayor riesgo de muerte. La edad avanzada (1-3), la obesidad, la diabetes y la hipertensión $(1,4,5)$ son factores de riesgo para la progresión a etapas graves de la enfermedad. Sin embargo, se conoce poco sobre el papel de los factores sociales.

La epidemia de COVID-19 adoptará especificidades de acuerdo con el estado de salud de las poblaciones, así como con su desarrollo económico y sus sistemas de salud. La desigualdad social es una característica de la sociedad mexicana y otros países de América Latina, que se expresa en la concentración del ingreso en una minoría al tiempo que una proporción

\footnotetext{
1 Universidad Autónoma Metropolitana unidad Xochimilco, Ciudad de México, México. ORCID http://orcid.org/0000-0002-5870-1729 $₫$ Luis OrtizHernández, lortiz@correo.xoc.uam.mx
} 
importante de la población vive en la pobreza (6). Las disparidades no sólo se presentan entre individuos y familias; también existen disparidades espaciales. México está polarizado geográficamente; en la región sur las tasas de pobreza e informalidad son altas, más población vive en áreas rurales y existe menor disponibilidad de establecimientos médicos (7). Otra característica de la sociedad mexicana es el racismo, que se expresa en desventajas de las personas con ascendencia indígena (8). El racismo se relaciona con las disparidades socioeconómicas, ya que entre las personas indígenas y con piel morena es más frecuente la pobreza o la pertenencia a niveles socioeconómicos más bajos, que predominan en los estados del sur (6,8). Lo anterior explica la mayor morbilidad por infecciones y mortalidad general en las personas indígenas (9).

En este contexto, el objetivo del estudio fue determinar si existen disparidades asociadas a la estratificación socioeconómica, la etnicidad, la región geográfica y los servicios médicos en la progresión de la COVID-19 en las personas mexicanas infectadas por SARS-CoV-2.

\section{MATERIAL Y MÉTODOS}

Se realizó un estudio observacional, retrospectivo y transversal para analizar los datos registrados por la Dirección General de Epidemiología de la Secretaría de Salud del Gobierno Federal de México respecto de los casos estudiados para detección de infección por SARS-CoV-2 (10). La base de datos contiene la información de los casos reportados ante la autoridad sanitaria en México hasta el 10 de julio del 2020. La información proviene de dos fuentes. La primera son los casos sospechosos o confirmados de COVID-19 reportados por cualquier establecimiento médico. En México, el 27 de febrero se solicitó a todos los establecimientos de atención médica (incluidos los tres niveles de atención de los sectores público y privado) que notificaran los casos de infección por SARS-CoV-2 (11). La segunda fuente son los casos detectados a través del Sistema de Vigilancia Epidemiológica de Enfermedad Respiratoria Viral (SISVER), que comenzó a funcionar el 5 de abril de 2020 (12). El SISVER opera a través de las unidades de salud monitoras de enfermedades respiratorias (USMER), que son 475 establecimientos distribuidos en todos los estados en los cuales se monitorea la existencia de 14 virus, incluido el SARS-CoV-2.

En la base de datos original existían 715295 registros de casos sospechosos, confirmados y negativos de infección por SARS-CoV-2. Se excluyeron los registros de los últimos 10 días para reducir un posible sesgo causado por el retraso en la notificación de los casos, quedando 587306 registros. Los casos confirmados eran 252 761. De las personas con un resultado positivo de SARS-CoV-2, el 95,8\% $(n=242309)$ presentaba datos completos. La variable con más datos faltantes fue la etnicidad $(3,3 \%)$. Al eliminar a los menores de 20 años, la muestra para el análisis fue de 234870 registros.

Para evaluar la severidad de la COVID-19 se analizaron cuatro desenlaces: neumonía, muerte, hospitalización y requerimiento de intubación o ingreso a la unidad de cuidados intensivos (Int-UCI). Estas variables eran dicotómicas.

Las instituciones donde se registró cada caso se clasificaron en tres categorías: seguridad social, dirigida a la "población abierta" (es decir, personas sin seguridad social) y servicios privados. Se calculó el número de días transcurridos entre el inicio de los síntomas y la atención recibida en los establecimientos de salud y entre el inicio de los síntomas y la fecha de corte de la información. Como parte de las variables sociodemográficas se consideró la pertenencia a un grupo indígena, el sexo y la edad.

En el SISVER se registra si las personas tienen las siguientes condiciones médicas: obesidad, hipertensión arterial, diabetes mellitus, asma, enfermedades cardiacas, inmunosupresión, insuficiencia renal crónica y enfermedad pulmonar obstructiva crónica. En un análisis factorial exploratorio se observó que la obesidad, la diabetes y la hipertensión se ubicaron en un mismo factor. Por lo anterior, se sumó el número de estas condiciones y la variable resultante se denominó "comorbilidades cardiometabólicas". Con este indicador se reconoce que, con frecuencia, las personas no presentan solo una condición cardiometabólica.

Para medir la posición socioeconómica de los individuos se vinculó a los datos del SISVER con la base de datos del Índice de Marginación Municipal del 2015 del Consejo Nacional de Población (CONAPO) $(13,14)$. Para construir este índice se utilizaron nueve variables sobre escolaridad, viviendas sin servicios básicos o en condiciones precarias, población en localidades rurales y ocupados con bajos ingresos. El rango de valores posibles del índice va de -2,2283 (baja marginación) a 5,0265 (alta marginación). En los casos registrados en el SISVER los percentiles 0, 25, 50, 75 y 100 del índice de marginación fueron -2,228, -1,722, $-1,527,-1,188$ y 4,845, respectivamente. De la base de datos de la CONAPO también se obtuvo la información del tamaño de población de los municipios donde residían las personas.

El análisis estadístico se realizó utilizando el programa Stata, versión 15 (Stata Corp., Texas, Estados Unidos). Se obtuvieron frecuencias relativas para las variables categóricas, mientras que para las variables continuas se calcularon medias. Se estimó la distribución de las características sociodemográficas y clínicas de acuerdo con cuartiles de marginación municipal. Se estimaron las prevalencias de los indicadores de severidad de COVID-19 de acuerdo con la marginación municipal, la etnicidad, la región geográfica, las características de la atención médica y las condiciones médicas. Para comparar proporciones se estimó la prueba de chi cuadrado y para contrastar medias se estimó la prueba de ANOVA.

Para cada indicador de progresión de la enfermedad se estimaron modelos multinivel de regresión de Poisson teniendo como variables independientes a la marginación municipal, la etnicidad, la región geográfica, las características sociodemográficas, las características de la atención médica y las condiciones médicas. En los modelos se incorporó una variable con deciles del índice de marginación municipal. La variable que identificaba a los municipios fue definida como intercepto aleatorio. Con los modelos multinivel se reconoce que las observaciones de personas que habitan en el mismo municipio no son independientes. A partir de los modelos se estimaron razones de prevalencias.

La primera versión de este manuscrito fue realizada con datos al corte del 28 de abril. Dados los cambios rápidos en la epidemia en México, se compararon los resultados con datos del 28 de abril con los del 10 de julio. Para ello, los mismos modelos multinivel se estimaron utilizando la base de datos con fecha de corte del 28 de abril. Después de aplicar los mismos criterios de exclusión de los datos descritos previamente, la base de esta última fecha contenía 10122 registros.

El proyecto fue aprobado por el Comité de Bioética para la Investigación en Seres Humanos del Centro de Investigación y de Estudios Avanzados del Instituto Politécnico Nacional. La confidencialidad de la información está garantizada ya que la base de datos no contiene información que permita identificar 
a las personas. De acuerdo con el Reglamento de la Ley General de Salud en Materia de Investigación para la Salud de México (artículo 17), el presente estudio puede ser clasificado como "sin riesgo" dado que se utilizó un diseño retrospectivo basado en registros existentes, por lo cual no se realizó ningún tipo de intervención. Por ello, puede prescindirse del consentimiento informado.

\section{RESULTADOS}

Poco más de la mitad de los casos correspondían a hombres, $1 \%$ era indígena y la mayoría de ellos fueron notificados en instituciones de seguridad social o para población abierta (cuadro 1). La condición médica de mayor prevalencia fue la hipertensión arterial, seguida de la obesidad y la diabetes. Del total de los casos, la tercera parte recibió atención hospitalaria, una cuarta parte desarrolló neumonía, 4\% requirió Int-UCI y el 14\% falleció.

Entre los casos que residían en los municipios con mayor marginación fueron mayores las proporciones de personas indígenas, que residían en la región sur y con más comorbilidades cardiometabólicas (cuadro 2). En municipios de mayor marginación, el porcentaje de casos notificados en instituciones para población abierta fue mayor, pero fue el más bajo en servicios privados.

Las prevalencias de los indicadores de gravedad de COVID19 fueron más altas en las personas indígenas, en las que

CUADRO 1. Características de los casos positivos de infección por SARS-CoV-2 en población mexicana, $2020(n=234$ 870)

\begin{tabular}{|c|c|}
\hline & $\%$ \\
\hline Hombres & 54,5 \\
\hline Indígenas & 1,0 \\
\hline \multicolumn{2}{|l|}{ Marginación municipal, cuartiles } \\
\hline I & 22,7 \\
\hline II & 26,3 \\
\hline III & 26,0 \\
\hline IV & 25,0 \\
\hline \multicolumn{2}{|l|}{ Institución } \\
\hline Seguridad social & 43,0 \\
\hline Población abierta & 54,1 \\
\hline Servicios privados & 2,9 \\
\hline \multicolumn{2}{|l|}{ Región } \\
\hline Norte & 22,5 \\
\hline Centro & 44,0 \\
\hline Occidente & 13,2 \\
\hline Sur & 20,3 \\
\hline \multicolumn{2}{|l|}{ Comorbilidades } \\
\hline Hipertensión arterial & 20,9 \\
\hline Obesidad & 19,9 \\
\hline Diabetes mellitus & 17,0 \\
\hline \multicolumn{2}{|l|}{ Desenlaces } \\
\hline Hospitalizados & 31,2 \\
\hline Neumonía & 24,2 \\
\hline Defunciones & 13,5 \\
\hline \multirow[t]{2}{*}{ Intubados o ingreso a unidad de cuidados intensivos } & 4,2 \\
\hline & $\bar{x}$ \\
\hline Edad (años) & 46,8 \\
\hline Tiempo para recibir atención (días) & 4,2 \\
\hline
\end{tabular}

\%, porcentaje; $\bar{x}$, media.
Fuente: estimaciones propias derivadas de datos oficiales (corte al 10 de julio, 2020).
CUADRO 2. Características de adultos mexicanos infectados por SARS-CoV-2 $(n=234870)$ de acuerdo con el nivel de marginación municipal

\begin{tabular}{|c|c|c|c|c|}
\hline & \multicolumn{4}{|c|}{ Cuartiles de marginación municipal } \\
\hline & I & $\overline{\text { II }}$ & III & IV \\
\hline & $\%$ & $\overline{\%}$ & $\%$ & $\%$ \\
\hline Hombre & 53,1 & 54,1 & 54,9 & 55,8 \\
\hline Indígenas & 0,4 & 0,5 & 0,7 & 2,6 \\
\hline \multicolumn{5}{|l|}{ Región } \\
\hline Norte & 22,3 & 26,6 & 30,5 & 10,0 \\
\hline Centro & 67,0 & 63,8 & 17,0 & 30,4 \\
\hline Occidente & 9,8 & 8,5 & 18,3 & 15,7 \\
\hline Sur & 0,9 & 1,1 & 34,2 & 43,9 \\
\hline \multicolumn{5}{|l|}{ Institución } \\
\hline Seguridad social & 44,1 & 45,5 & 46,3 & 35,9 \\
\hline Población abierta & 51,0 & 51,7 & 51,6 & 62,2 \\
\hline Servicios privados & 4,9 & 2,8 & 2,1 & 1,9 \\
\hline \multicolumn{5}{|l|}{ Comorbilidades cardiometabólicas } \\
\hline Ninguna & 62,1 & 60,9 & 58,9 & 57,5 \\
\hline Una & 24,3 & 25,1 & 26,1 & 27,0 \\
\hline \multirow[t]{2}{*}{ Dos o tres } & 13,6 & 14,0 & 15,0 & 15,5 \\
\hline & $\bar{x}$ & $\bar{x}$ & $\bar{x}$ & $\bar{x}$ \\
\hline Edad (años) & 47,0 & 46,7 & 46,1 & 47,1 \\
\hline Tiempo para recibir atención (días) & 4,1 & 4,2 & 4,2 & 4,3 \\
\hline
\end{tabular}

$\%$, porcentaje; $\bar{x}$, media.

Todas las diferencias fueron significativas $(p<0,001)$ utilizando la prueba de chi cuadrado (porcentajes) o ANOVA (medias).

Fuente: estimaciones propias derivadas de datos oficiales (corte al 10 de julio, 2020).

residían en municipios con mayor marginación, entre quienes tardaron más en recibir atención médica y entre quienes tenían más comorbilidades cardiometabólicas (cuadro 3). Los individuos que contaban con seguridad social tuvieron la prevalencia más alta de neumonía, defunciones y hospitalización, pero la más baja de Int-UCI. Quienes usaron servicios privados tuvieron la prevalencia más baja de defunciones, pero la mayor de Int-UCI. Entre las personas de la región centro existieron las proporciones más altas de neumonía, defunciones y hospitalización, mientras que en las de la región sur fue más alta la de Int-UCI y ocupó el segundo lugar en defunciones.

Considerando los resultados del último corte (10 de julio), se observó que vivir en un municipio con mayor marginación se relacionó con mayor riesgo de neumonía, defunción (cuadro 4), hospitalización y muerte (cuadro 5). Este riesgo se mantuvo después de ajustar por otras características sociales, demográficas y clínicas. Las personas indígenas tuvieron mayor riesgo de neumonía, muerte y hospitalización, y dicho riesgo se hizo más fuerte después de ajustar por otras variables. La etnicidad no se asoció con la probabilidad de requerir Int-UCI. En comparación con las personas atendidas en los servicios de la seguridad social, las que fueron atendidas en servicios para población abierta tuvieron menor riesgo de neumonía, defunciones y hospitalización, pero mayor probabilidad de requerir Int-UCI. El acudir a servicios privados se relacionó con mayor probabilidad de neumonía y necesidad de Int-UCI, pero menor probabilidad de morir. A medida que aumentó el tiempo en recibir atención médica, fue mayor el riesgo de neumonía e Int-UCI, pero menor el de muerte. En comparación con las personas que residían en la región norte, las que vivían en las regiones centro y sur tuvieron mayor riesgo de los cuatro desenlaces. Las personas de la 
CUADRO 3. Prevalencia de los indicadores de gravedad de acuerdo con características sociodemográficas y clínicas en adultos mexicanos infectados por SARS-CoV2

\begin{tabular}{|c|c|c|c|c|}
\hline & Neumonía & Defunción & Hospitalización & Int-UCI \\
\hline & $\%$ & $\%$ & $\%$ & $\%$ \\
\hline \multicolumn{5}{|l|}{ Indígena } \\
\hline No & 24,1 & 13,4 & 31,2 & 4,2 \\
\hline Sí & 34,9 & 19,9 & 39,6 & 7,0 \\
\hline \multicolumn{5}{|c|}{ Marginación municipal (cuartiles) } \\
\hline I & 22,0 & 12,1 & 28,5 & 3,7 \\
\hline II & 25,9 & 14,1 & 32,4 & 3,7 \\
\hline III & 22,0 & 13,3 & 30,6 & 4,3 \\
\hline IV & 26,7 & 14,3 & 33,3 & 5,2 \\
\hline \multicolumn{5}{|l|}{ Región } \\
\hline Norte & 21,4 & 12,8 & 28,6 & 3,9 \\
\hline Centro & 28,8 & 14,8 & 34,6 & 4,3 \\
\hline Occidente & 17,3 & 9,5 & 25,9 & 3,3 \\
\hline Sur & 21,9 & 13,9 & 30,2 & 5,0 \\
\hline \multicolumn{5}{|l|}{ Institución } \\
\hline Seguridad social & 29,9 & 20,6 & 43,8 & 3,1 \\
\hline Población abierta & 20,4 & 8,2 & 21,3 & 4,8 \\
\hline Servicios privados & 28,2 & 6,3 & 31,1 & 9,9 \\
\hline \multicolumn{5}{|c|}{ Tiempo para recibir atención (terciles) } \\
\hline I & 21,7 & 13,9 & 32,0 & 3,1 \\
\hline ॥ & 21,6 & 11,2 & 26,1 & 3,9 \\
\hline III & 28,1 & 14,6 & 34,0 & 5,5 \\
\hline \multicolumn{5}{|c|}{ Comorbilidades cardiometabólicas } \\
\hline Ninguna & 16,7 & 7,6 & 21,6 & 2,5 \\
\hline Una & 31,2 & 18,0 & 39,6 & 5,9 \\
\hline Dos o tres & 43,3 & 29,6 & 56,1 & 8,6 \\
\hline
\end{tabular}

$\%$, porcentaje; Int-UCl, intubación o ingreso a unidad de cuidados intensivos. Todas las diferencias fueron significativas $(p<0,001)$ utilizando la prueba de chi cuadrado. Fuente: estimaciones propias derivadas de datos oficiales (corte al 10 de julio, 2020).

CUADRO 4. Modelos de regresión multinivel de Poisson considerando los indicadores de progresión de COVID como variables dependientes en mexicanos infectados por SARSCoV-2, 2020

\begin{tabular}{|c|c|c|c|c|c|c|}
\hline & \multicolumn{3}{|c|}{ Neumonía } & \multicolumn{3}{|c|}{ Defunción } \\
\hline & \multirow{2}{*}{$\begin{array}{c}\text { 28-abril } \\
\text { M2 }\end{array}$} & \multicolumn{2}{|c|}{ 10-julio } & \multirow{2}{*}{$\begin{array}{c}\text { 28-abril } \\
\text { M2 }\end{array}$} & \multicolumn{2}{|c|}{ 10-julio } \\
\hline & & M1 & M2 & & M1 & M2 \\
\hline & $\mathrm{RP}$ & $\mathrm{RP}$ & $\mathrm{RP}$ & $\mathrm{RP}$ & $\mathrm{RP}$ & $\mathrm{RP}$ \\
\hline Indígenas & $1,49^{b}$ & $1,19^{c}$ & $1,22^{\mathrm{c}}$ & $1,57^{\mathrm{a}}$ & $1,14^{\mathrm{b}}$ & $1,23^{c}$ \\
\hline Índice de marginación, deciles & $1,07^{c}$ & $1,05^{c}$ & $1,06^{c}$ & $1,08^{c}$ & $1,04^{c}$ & $1,06^{c}$ \\
\hline \multicolumn{7}{|l|}{ Región } \\
\hline Centro & $1,35^{\mathrm{c}}$ & $1,89^{c}$ & $1,82^{c}$ & 0,90 & $1,54^{c}$ & $1,52^{c}$ \\
\hline Occidente & 0,86 & $1,25^{c}$ & $1,17^{\mathrm{b}}$ & 0,88 & 1,05 & 1,01 \\
\hline Sur & 1,11 & $1,39^{c}$ & $1,23^{c}$ & 0,98 & $1,37^{c}$ & $1,19^{b}$ \\
\hline \multicolumn{7}{|l|}{ Institución } \\
\hline Población abierta & 1,01 & $0,81^{\circ}$ & $0,82^{\mathrm{c}}$ & 0,90 & $0,51^{\mathrm{c}}$ & $0,52^{c}$ \\
\hline Servicios privados & $1,42^{c}$ & $1,30^{\circ}$ & $1,34^{c}$ & $0,33^{c}$ & $0,38^{\mathrm{c}}$ & $0,40^{c}$ \\
\hline Tiempo para recibir atención & $1,03^{c}$ & $1,02^{\mathrm{c}}$ & $1,02^{\mathrm{c}}$ & $0,98^{\mathrm{a}}$ & $0,99^{c}$ & $0,99^{a}$ \\
\hline Comorbilidades cardiometabólicas & $1,24^{c}$ & $1,24^{c}$ & $1,23^{c}$ & $1,40^{c}$ & $1,31^{\mathrm{c}}$ & $1,29 c$ \\
\hline
\end{tabular}


CUADRO 5. Modelos de regresión multinivel de Poisson considerando los indicadores de progresión de COVID como variables dependientes en mexicanos infectados por SARSCoV-2, 2020

\begin{tabular}{|c|c|c|c|c|c|c|}
\hline & \multicolumn{3}{|c|}{ Hospitalización } & \multicolumn{3}{|c|}{$\begin{array}{l}\text { Intubación o ingreso a unidad de } \\
\text { cuidados intensivos }\end{array}$} \\
\hline & \multirow{2}{*}{$\frac{\text { 28-abril }}{\mathrm{M} 2}$} & \multicolumn{2}{|c|}{ 10-julio } & \multirow{2}{*}{$\frac{28 \text {-abril }}{\mathrm{M} 2}$} & \multicolumn{2}{|c|}{ 10-julio } \\
\hline & & M1 & $\mathrm{M} 2$ & & M1 & $\mathrm{M} 2$ \\
\hline & $\mathrm{RP}$ & $\mathrm{RP}$ & $\mathrm{RP}$ & $\mathrm{RP}$ & $\mathrm{RP}$ & $\mathrm{RP}$ \\
\hline Indígenas & 1,22 & $1,07^{a}$ & $1,14^{a}$ & 1,04 & 1,14 & 1,06 \\
\hline Índice de marginación, deciles & $1,06^{c}$ & $1,04^{c}$ & $1,06^{c}$ & $1,11^{c}$ & $1,08^{c}$ & $1,05^{b}$ \\
\hline \multicolumn{7}{|l|}{ Región } \\
\hline Centro & $1,32^{c}$ & $1,58^{c}$ & $1,54^{c}$ & $1,41^{\mathrm{a}}$ & $1,50^{c}$ & $1,43^{c}$ \\
\hline Occidente & 0,88 & $1,15^{b}$ & $1,10^{\mathrm{a}}$ & 0,87 & $1,23^{\mathrm{a}}$ & 1,13 \\
\hline Sur & 1,08 & $1,37^{c}$ & $1,20^{\mathrm{c}}$ & $1,55^{\mathrm{a}}$ & $1,46^{c}$ & $1,38^{\mathrm{c}}$ \\
\hline \multicolumn{7}{|l|}{ Institución } \\
\hline Población abierta & $0,72^{\mathrm{c}}$ & $0,58^{c}$ & $0,58^{c}$ & $3,19^{c}$ & $2,08^{c}$ & $2,10^{c}$ \\
\hline Servicios privados & 0,99 & $0,94^{\mathrm{a}}$ & 0,97 & $4,41^{c}$ & $4,31^{c}$ & $4,45^{c}$ \\
\hline Tiempo para recibir atención & 1,00 & 1,00 & 1,00 & $1,04^{c}$ & $1,05^{c}$ & $1,05^{c}$ \\
\hline Comorbilidades cardiometabólicas & $1,20^{c}$ & $1,22^{\mathrm{c}}$ & $1,21^{\mathrm{c}}$ & $1,30^{c}$ & $1,34^{c}$ & $1,37^{c}$ \\
\hline
\end{tabular}

región occidental tuvieron mayor riesgo de neumonía y hospitalización. Las condiciones cardiometabólicas se relacionaron con mayor riesgo de los cuatro indicadores de gravedad.

Al comparar las estimaciones obtenidas con las dos fechas de corte de los datos (cuadros 3 y 4), todas las asociaciones observadas en el primer momento ( 28 de abril) se mantuvieron en el segundo (10 de julio), aunque en algunos casos se modificó la fuerza de asociación. Con los datos del 10 de julio fueron menores las diferencias en el riesgo en neumonía y defunción de acuerdo con la etnicidad, la marginación municipal, el uso de servicios privados y el tiempo para recibir atención médica. Las diferencias en la probabilidad de requerir Int-UCI de acuerdo con la marginación municipal también disminuyeron. Por otra parte, algunas asociaciones emergieron con los datos más recientes. El mayor riesgo de hospitalización en las personas indígenas se hizo significativo. También comenzó a observarse menor riesgo de neumonía y defunción en la población abierta; mayor riesgo de neumonía y hospitalización en las personas de las regiones occidental y sur, y mayor probabilidad de muerte en aquellas de las regiones centro y sur.

\section{DISCUSIÓN}

De acuerdo con la epidemiología social, los grupos sociales en desventaja presentan mayor riesgo de enfermar y morir, ya que por las características de su entorno tienen mayor exposición a factores de riesgo al tiempo que tienen menos factores protectores o recursos para hacer frente a las enfermedades (15). Siguiendo este planteamiento, el hallazgo central de este estudio es que la marginación municipal es un predictor de presentar formas graves de COVID-19 y esta asociación fue independiente de otros predictores bien establecidos como la edad y las comorbilidades cardiometabólicas.

Entre los casos de infección por SARS-CoV-2 de México se observó que conforme la marginación municipal fue mayor, fueron más altas las proporciones de personas indígenas, que residían en la región sur, con comorbilidades y que tardaron más en recibir atención. Sin embargo, la asociación entre la marginación municipal y las formas graves de COVID-19 no se modificó después de ajustar por estas variables. Eso implica que existen otros procesos o mecanismos que explican las disparidades socioeconómicas en la progresión de la COVID-19. Por ejemplo, las experiencias de privación y subordinación son estresores crónicos que se experimentan con más frecuencia conforme la posición socioeconómica es más baja (16). Las concentraciones elevadas de cortisol (que son parte de la respuesta fisiológica al estrés crónico) se relacionan con mayor riesgo de mortalidad por COVID-19 (17). A su vez, los estresores tienen efectos deletéreos en la función inmunitaria, están asociados con marcadores de inflamación (18) y contribuyen a desregular los mecanismos de coagulación (19), aspectos todos involucrados en la fisiopatología de la COVID-19 (3,20).

Tanto ser indígena como vivir en la región sur se relacionaron con la gravedad de la COVID-19. Estas disparidades basadas en la localización geográfica y la etnicidad están íntimamente vinculadas con la desigualdad socioeconómica: en la región sur existen tasas más altas de pobreza y se concentra la población indígena (6), lo que muestra como se intersectan diferentes formas de desigualdad. Vivir en la región más empobrecida del país puede implicar un efecto que es independiente a otros factores; por ejemplo, a través de la menor disponibilidad de servicios médicos de calidad (7). Es de notar que las personas indígenas tuvieron mayor riesgo de neumonía y muerte; sin embargo, no existieron diferencias basadas en la etnicidad respecto de la necesidad de Int-UCI. Estos resultados podrían implicar que las personas indígenas, a pesar de tener una salud precaria, no siempre tuvieron mayor acceso a la atención que su condición requería. Además de ser pobre, el ser indígena en una sociedad racializada como la mexicana también puede implicar dificultades culturales y lingüísticas o recibir un trato discriminatorio en los servicios de salud. En Estados Unidos de América (otra sociedad racializada) las minorías étnicas (afroamericanos 
e hispanos) también han estado en mayor riesgo de desarrollar formas graves de COVID-19 (21).

Las personas que utilizaron servicios privados tuvieron menor riesgo de muerte, pero mayor probabilidad de requerir Int-UCI. Este menor riesgo de progresión de la COVID-19 en los servicios privados podría atribuirse a su mayor calidad (y consecuente mayor costo). Es decir, es posible que el mayor soporte de vida utilizado en los servicios privados explique la menor mortalidad por COVID-19. Desde inicios del siglo, en México la calidad de los servicios privados ha sido evaluada de forma positiva por la población, ya que en ellos los tiempos de espera son menores y se percibe que existe más probabilidad de que los problemas de salud se resuelvan $(22,23)$. En contrapartida, históricamente los recursos con que cuentan los servicios públicos han sido menores a los requeridos dada su demanda de atención (24). En el contexto de la epidemia de COVID-19, esto ha implicado que los servicios públicos han llegado a saturarse a pesar de los esfuerzos gubernamentales (25). De este modo, el problema radica en que la atención a la salud en México se ha mercantilizado y eso implica que pocos tienen acceso a servicios de calidad, pues el acceso a ellos depende de la capacidad adquisitiva de las personas. El gobierno de México ha propuesto la integración de un sistema de salud único para reducir las barreras para el acceso. Para que esto sea alcanzado con éxito deberá garantizarse que la calidad de los servicios públicos sea tan eficaz como parecen ser los privados.

Las condiciones cardiometabólicas son fuertes predictores de la progresión de la COVID-19 (1,4,5), lo que también fue observado en la población mexicana. Además, en México, conforme disminuye la posición socioeconómica es más probable tener hipertensión, diabetes y obesidad (15). Aunque encontramos que las comorbilidades cardiometabólicas fueron más frecuentes en las personas con menor posición socioeconómica, en los modelos de regresión estas no modificaron la relación de la marginación municipal con la progresión de la COVID-19. Hasta ahora se ha subrayado que la vulnerabilidad proviene de las condiciones médicas preexistentes; además de ello, se requiere evidenciar que también existe vulnerabilidad social.

El análisis de los datos en dos momentos (28 de abril y 10 de julio) muestra que desde las fases tempranas de la epidemia en México se presentaron disparidades sociales en la progresión de la COVID-19 y es probable que estas se mantengan. Estas disparidades asociadas a la posición socioeconómica, la etnicidad, la región y el sector de los servicios muestran que los efectos de la infección no sólo se deben a las características biológicas del SARS-CoV-2, sino que tienen patrones de distribución de acuerdo con factores sociales. Es fundamental considerar que, ante un mismo problema de salud, los recursos (o la falta de ellos) para afrontarlo dependen en gran medida de la posición de las personas en la sociedad. Así, las consecuencias de la epidemia no sólo deben pensarse como producto de un nuevo agente infeccioso, sino como la interacción de este con las estructuras sociales de cada país.

Los resultados de nuestro estudio deben ponderarse en función de sus fortalezas y limitaciones. A diferencia de reportes previos elaborados con muestras hospitalarias (1-5), nuestro análisis se basó en los casos de infección por coronavirus confirmados en México, con lo cual aumenta la posibilidad de extrapolar nuestros resultados. Respecto a las limitaciones, existe un sesgo de selección, ya que desde el 24 de marzo se promovió que las personas asintomáticas o con síntomas leves se mantuvieran en casa y las prevalencias estimadas deben ser mayores a las reales. Para reducir el efecto de este sesgo, incluimos en los modelos el tiempo en que iniciaron los síntomas y el cierre del registro de casos, con lo cual se distingue si los casos se presentaron al inicio o en etapas posteriores de la epidemia. La medición de la posición socioeconómica que utilizamos no es perfecta, ya que asignamos las características sociales del municipio de residencia a las personas infectadas por SARS-CoV-2 y puede no existir correspondencia entre la posición socioeconómica de las personas y la del municipio. Al mismo tiempo, la posición socioeconómica de la localidad puede tener impacto en la salud de las personas a través de factores como la disponibilidad de servicios o la existencia de ambientes sociales o físicos adversos (26). La medición de la etnicidad también es una limitación, ya que no son claros los criterios que utilizan los médicos para definir cuando una persona es indígena; quizá debido a esto la etnicidad es la variable con más datos faltantes. En México, la proporción de población indígena puede variar según se utilice el criterio del habla de lengua indígena $(8,3-8,9 \%)$ o el que las personas se autoadscriban a un grupo indígena (14,0-16,0\%) (27). Se puede especular, entonces, que la baja frecuencia de indígenas en los registros de infectados por SARS-CoV-2 de México (1\%) puede ser resultado de que los médicos utilizan el criterio lingüístico. Sin embargo, las personas que se autoadscriben a una identidad indígena también se enfrentan a desventajas sociales (27).

En síntesis, en México tener menor posición socioeconómica, ser indígena y vivir en los estados del sur se relacionó con mayor riesgo de presentar formas severas de COVID19; por el contrario, el uso de servicios privados se asoció con menor mortalidad. Después de considerar otras covariables, estas relaciones se mantuvieron. Nuestros resultados muestran que, para dar una mejor respuesta a la epidemia de COVID-19 se requiere un enfoque de equidad en salud en la asignación de recursos, equipamiento y personal. Los grupos y las localidades de baja posición socioeconómica (muchas de ellas en el sur, donde predomina la población indígena) deben ser considerados prioritarios. Pero esta prioridad no sólo debe darse en medidas puntuales para reducir el contagio o la progresión de la COVID19; también deben implementarse políticas de redistribución de los recursos que permitan erradicar las disparidades sociales. Otra implicación de nuestro estudio es que se requiere mejorar el monitoreo de las desigualdades en salud, para lo cual es necesario incluir el registro de variables como la escolaridad de las personas. Finalmente, en otros países de América Latina existe una proporción significativa de población indígena (28), además de que la región se caracteriza por la desigualdad socioeconómica. Por ello, puede ser pertinente verificar si las disparidades aquí documentadas se reproducen en otras sociedades.

Contribución de los autores. LOH concibió el estudio original y planificó el análisis de los datos. MPS analizó los datos. LOH y MPS interpretaron los resultados, escribieron el manuscrito y lo revisaron. Los dos autores revisaron y aprobaron la versión final.

\section{Conflictos de intereses. Ninguno declarado.}

Declaración. Las opiniones expresadas en este manuscrito son únicamente responsabilidad de los autores y no reflejan necesariamente las de la Revista Panamericana de Salud Pública o la Organización Panamericana de la Salud. 


\section{REFERENCIAS}

1. Li B, Yang J, Zhao F, Zhi L, Wang X, Liu L, et al. Prevalence and impact of cardiovascular metabolic diseases on COVID-19 in China. Clin Res Cardiol. 2020;109(5):531-8.

2. Myers L, Parodi S, Escobar G, Liu V. Characteristics of hospitalized adults with COVID-19 in an integrated health care system in California [letter]. JAMA. 2020; 323(21):2195-2198.

3. Huang C, Wang Y, Li X, Ren L, Zhao J, Hu Y, et al. Clinical features of patients infected with 2019 novel coronavirus in Wuhan, China. Lancet. 2020;395(10223):497-506.

4. Singh A, Gupta R, Misra A. Comorbidities in COVID-19: Outcomes in hypertensive cohort and controversies with renin angiotensin system blockers. Diabetes Metab Syndr. 2020;14(4):283-7.

5. Guan WJ, Ni ZY, Hu Y, Liang WH, Ou CQ, He JX, et al. Clinical characteristics of coronavirus disease 2019 in China. N Engl J Med. 2020;382(18):1708-20

6. Consejo Nacional de Evaluación de la Política de Desarrollo Social. Informe de evaluación de la política de desarrollo social 2018. Ciudad de México: CONEVAL; 2018.

7. Organization for Economic Co-operation and Development. Measuring well-being in Mexican states. Paris: Organization for Economic Co-operation and Development Publishing; 2015.

8. Ortiz-Hernandez L, Ayala-Guzman C, Perez-Salgado D. Posición socioeconómica, discriminación y color de piel en México. Perfiles Latinoam. 2018;26(51):215-39.

9. Juarez-Ramirez C, Marquez-Serrano M, Salgado de Snyder N, Pelcastre-Villafuerte BE, Ruelas-Gonzalez MG, Reyes-Morales $\mathrm{H}$. La desigualdad en salud de grupos vulnerables de México: adultos mayores, indígenas y migrantes. Rev Panam Salud Publica. 2014;35(4):284-90.

10. Secretaría de Salud, Gobierno de México. Datos abiertos - Bases Históricas [Internet]. En: Dirección General de Epidemiología. México. 2020 [consultado 28 abril y 10 de julio, 2020]. Disponible en: https://www.gob.mx/salud/documentos/datos-abiertos-bases -historicas-direccion-general-de-epidemiologia.

11. Secretaría de Salud, Gobierno de México. Aviso epidemiológico CONAVE /04/2020/COVID-19. Enfermedad COVID-19 por SARS-CoV-2 (febrero 27). En: Comité Nacional para la Vigilancia Epidemiológica (CONAVE). México. 2020.

12. Secretaría de salud, Gobierno de México. Aviso epidemiológico CONAVE /09/2020/COVID-19. Enfermedad COVID-19 por SARS-CoV-2 (abril, 6). En: Comité Nacional para la Vigilancia Epidemiológica (CONAVE). México; 2020.

13. Consejo Nacional de Población. Datos abiertos del índice de marginación [Internet]. Consejo Nacional de Población. 2016 [consultado el 20 abril 2020]. Disponible en: http://www.conapo.gob.mx/es/ CONAPO/Datos_Abiertos_del_Indice_de_Marginacion.

14. Consejo Nacional de Población. Índice de marginación por entidad federativa y municipio 2015. Ciudad de México: Consejo Nacional de Población 2016.
15. Ortiz-Hernández L, Pérez-Salgado D, Tamez-González S. Desigualdad socioeconómica y salud en México. Rev Med Inst Mex Seguro Soc. 2015;53(3):336-47.

16. Marmot M. Status syndrome A challenge to Medicine. JAMA. 2006;295(11).

17. Tan T, Khoo B, Mills EG, Phylactou M, Patel B, Eng PC, et al. Association between high serum total cortisol concentrations and mortality from COVID-19. Lancet Diabetes Endocrinol. 2020 (ahead of print)

18. Aiello AE, Kaplan GA. Socioeconomic position and inflammatory and immune biomarkers of cardiovascular disease: applications to the Panel Study of Income Dynamics. Biodemography Soc Biol. 2009;55(2):178-205.

19. Tabassum F, Kumari M, Rumley A, Power C, Strachan D, Lowe G. Life course social position and d-dimer; findings from the 1958 British Birth Cohort. PloS One. 2014;9(5).

20. Tang N, Li D, Wang X, Sun Z. Abnormal coagulation parameters are associated with poor prognosis in patients with novel coronavirus pneumonia. J Thromb Haemost. 2020;18(4):844-7.

21. Yancy CW. COVID-19 and African Americans. JAMA. 2020; 323(19):1891-1892.

22. Ávila L, Wirtz V, Serván E, Bautista S, Barroso, González M. Utilización de servicios de salud. In: Gutiérrez J, Rivera-Dommarco J, Shamah-Levy T, Villalpando-Hernández S, Franco A, Cuevas-Nasu L, et al., editors. Encuesta Nacional de Salud y Nutrición 2012 Resultados Nacionales. Cuernavaca, México: Instituto Nacional de Salud Pública; 2012.

23. Puig A, Pagán J, Wong R. Assessing quality across healthcare subsystems in Mexico. J Ambul Care Manage. 2009;32(2):123-31.

24. Laurell A. Impacto del Seguro Popular en el sistema de salud mexicano. Ciudad Autónoma de Buenos Aires. CLACSO; 2013.

25. Cruz Á. Están llenos hospitales Covid en el Valle de México. La Jornada. 2020 martes, 12 de mayo, 2020, sección Política.

26. Meijer M, Röhl J, Bloomfield K, Grittner U. Do neighborhoods affect individual mortality? A systematic review and meta-analysis of multilevel studies. Soc Sci Med. 2012;74(8):1204-12.

27. Villarreal A. Ethnic Identification and its Consequences for Measuring Inequality in Mexico. Am Sociol Rev. 2014;79(4):775-806.

28. Montenegro RA, Stephens C. Indigenous health in Latin America and the Caribbean. Lancet. 2006;367(9525):1859-69.

Manuscrito recibido el 14 de mayo de 2020. Aceptado para su publicación, tras revisión, el 17 de julio de 2020. 


\section{Social inequalities in the progression of COVID-19 in the Mexican population}

ABSTRACT Objective. To determine if there are disparities associated with socioeconomic stratification, ethnicity, medical services, and geographic region in the progression of coronavirus disease (COVID-19) in Mexican adults infected with SARS-CoV-2.

Method. We analyzed data registered by the General Direction of Epidemiology of the Ministry of Health of the Federal Government of Mexico regarding the confirmed cases of SARS-CoV-2 infection. The analysis was limited to data from adults 20 years and older recorded up to July 10, $2020(n=234870)$. Indicators of severity of COVID-19 were hospitalization, development of pneumonia, requirement for intubation or admission to the intensive care unit, and death. Differences were estimated according to the level of municipal marginalization, belonging to an indigenous group, geographic region, and service sector. Prevalence ratios (PR) were estimated using multilevel regression models.

Results. People who lived in municipalities with greater marginalization were at greater risk of presenting the four indicators of severe forms of COVID-19 (PR=1.05 or 1.06). Indigenous people were at greater risk of pneumonia $(P R=1.22)$, hospitalization $(P R=1.14)$ and death $(P R=1.23)$. Among the cases treated in the private health sector, the risk of death was lower $(P R=0.40)$, but the use of intubation or admission to the intensive care unit was higher ( $P R=4.45)$.

Conclusions. The trends observed indicate that the effects of COVID-19 are not only related to the biological characteristics of SARS-CoV-2, but also to the resources (or lack thereof) to deal with it, which are distributed by social processes. 